\title{
Diabetischer Fuß muss nicht in die Druckkammer
}

\author{
Die hyperbare Sauerstofftherapie wird in Deutschland auch zur Behandlung diabetischer \\ Fußulzera eingesetzt. Eine neue Studie kann keinerlei positive Effekte ans Licht bringen.
}

\begin{abstract}
_ Seit mehr als 20 Jahren wird die hyperbare Sauerstofftherapie (HBO) bei diabetischen Fußulzera eingesetzt. Die Patienten begeben sich dafür in eine Überdruckkammer und atmen reinen Sauerstoff. Der Nutzen ist umstritten.

Für eine doppelblinde, randomisierte Studie wurden Patienten mit Fußulzera der Wagner-Stadien 2 bis 4 rekrutiert. Zusätzlich zur Standardtherapie erhielten sie entweder $30 \mathrm{HBO}$-Sitzungen von 90 Minuten Dauer oder ebenso viele Placebo-Sitzungen in der gleichen Kammer mit normaler Luft und Normaldruck. Primäres Studienziel war die Indikation für eine Amputation nach 12 Wochen. Als sekundäres Studienziel wurde die Wundheilung beurteilt.

107 Patienten wurden randomisiert, 103 schlossen die Studie ab. Eine Amputation war am Ende bei 13 der 54 Teil-
\end{abstract}

nehmer im Placeboarm und bei 11 der 49 Patienten mit HBO angezeigt (Odds Ratio 0,91, 95\%-Konfidenzintervall [KI]: $0,37-2,28, \mathrm{p}=0,846)$. Abgeheilt waren 12 Ulzera im Placeboarm und 10 mit der HBO-Therapie (Odds Ratio 0,90, KI: $0,35-2,31, \mathrm{p}=0,823)$. Auch sonst gab es keine statistischen Unterschiede.

Die Autoren diskutieren auch eine ältere Studie, in der ein Erfolg bei der Abheilung von diabetischen Ulzera beobachtet werden konnte [Löndahl $\mathrm{M}$ et al. Diabetes Care 2010;33:998-1003]. Diese hatte im Placeboarm hyperbare Raumluft verwendet, was die Durchblutung geschädigt haben könnte. Diesen Fehler wollten die Autoren nicht machen.

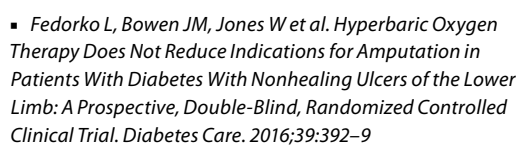

- Fedorko L, Bowen JM, Jones Wet al. Hyperbaric Oxygen Therapy Does Not Reduce Indications for Amputation in Patients With Diabetes With Nonhealing Ulcers of the Lower Limb: A Prospective, Double-Blind, Randomized Controlled Clinical Trial. Diabetes Care. 2016;39:392-9

\begin{abstract}
KOMMENTAR
Nun besteht wirklich kein Grund mehr, die $H B O$ anzuwenden. Schon bisher sprach nur eine Studie mit akzeptablem Design dafür, nämlich die von den Autoren kritisierte Arbeit von Löndahl et al. - und auch sie zeigte nur eine Beschleunigung der Ulkusabheilung, aber keine Verringerung der Amputationen. Außerdem heilten in dieser Studie nur ausreichend durchblutete Ulzera mit $\mathrm{HBO}$ besser, während bei ischämischen Ulzera kein Effekt nachzuweisen war [Löndahl M et al. Diabetologia. 2011;54:65-68]. Bei Verwendung von HBO in der täglichen Praxis können sogar negative Folgen resultieren [Margolis DJ et al. Diabetes Care. 2013;36:1961-6]. Es gibt wesentlich kostengünstigere, belegt wirksame Therapien, z. B. den Total Contact Cast. Ein breiter Einsatz wird allerdings durch die fehlende Vergütung verhindert.
\end{abstract}

Prof. Dr. med. M. Spraul

\section{Übersterblichkeit bei Diabetikern kein generelles Phänomen} \begin{abstract}
diovaskulärer Ursachen unter Diabetikern erhöht. Für Typ2-Diabetiker wurde das aber nie systematisch untersucht. Nun wurden Daten aus schwedischen Patientenregistern ausgewertet. Die 435.369 zwischen 1998 und 2011 registrierten Typ-2-Diabetiker wurden 2.117.483 alters-, geschlechts- und herkunftsgleichen Personen aus der Allgemeinbevölkerung gegenübergestellt. Dabei ergab sich, dass innerhalb von 4,7 Jahren $17,7 \%$ der Diabetiker und $14,5 \%$ der Kontrollpersonen starben. Die Raten für die kardiovaskuläre Mortalität betrugen $7,9 \%$ bzw. $6,1 \%$.
\end{abstract}

Laut Lehrmeinung sind Gesamtmortalität und Sterblichkeit infolge kar-

Die Übersterblichkeit von Diabetikern, also die absolute Differenz, erreicht damit ein historisches Tief von ca. $20 \%$. Die Autoren führen dies auf die Therapie mit Antidiabetika zurück, aber auch auf Lipidsenker und Antihypertensiva, die bei Diabetikern häufig aggressiver eingesetzt werden als bei Nichtdiabetikern.
Diese Gesamtwerte verdecken aber eine große Heterogenität. Diabetiker unter 55 Jahren hatten auch bei guter Stoffwechselkontrolle $\left(\mathrm{HbA}_{1 \mathrm{c}}\right.$-Wert

$<6,9 \%$ ) ein um $92 \%$ erhöhtes Gesamtsterberisiko. Zeigten sie noch keine Anzeichen einer Nephropathie, war das Risiko immerhin noch um $60 \%$ erhöht. Ganz anders sah es bei den alten Diabetikern über 75 Jahren aus. Hier hatten gut eingestellte Diabetiker ein um 5\% erniedrigtes Sterberisiko, Diabetiker ohne Nephropathie lagen sogar $14 \%$ unter den Kontrollpersonen.

Diese Ergebnisse rütteln an der verbreiteten Ansicht, die allen Diabetikern pauschal eine Übersterblichkeit zuschreibt. Dies ist eher bei jungen Patienten der Fall. Wer mit 75 noch lebt und gut eingestellt ist, trägt offenbar kein höheres Sterberisiko als die Gesamtbevölkerung.

Prof. Dr. med. H. Holzgreve

- TancrediM, Rosengren A, Svensson AM etal. Excess Mortality among Persons with Type 2 Diabetes. New Engl J Med. 2015;373:1720-32

Gut eingestellte ältere Patienten können lange leben. 